\title{
Recent Results and Future Prospects of Neutrino Oscillation Experiments
}

\author{
Roger A. Wendell ${ }^{* \dagger}$ \\ Departments of Physics, Kyoto University, Kyoto, Kyoto 606-8502, Japan \\ E-mail: rawescphys.kyoto-u.ac.jp
}

\begin{abstract}
Since the discovery of non-zero $\theta_{13}$ and oscillation-induced electron neutrino appearance, it has become possible to address the question of $\mathrm{CP}$ violation in neutrino oscillations in long-baseline experiments. Though current experiments have already begun to constrain $\delta_{c p}$, the parameter responsible for $\mathrm{CP}$ violation in the PMNS mixing paradigm, uncertainties in other oscillation parameters remain an obstacle to a precision measurement. This talk will review recent results in neutrino oscillation measurements, with a focus on progress towards and prospects for an observation (or rejection) of CP violation in neutrinos. Though emphasis will be placed on the long-baseline and atmospheric neutrino programs in Asia, discussion of results from worldwide experiments will be included.
\end{abstract}

The 3rd International Symposium on "Quest for the Origin of Particles and the Universe"

5-7 January 2017

Nagoya University, Japan

\footnotetext{
* Speaker.

${ }^{\dagger}$ The author gratefully acknowledges the support of the KMI2017 organizing committee for the opportunity to present at the symposium.
} 
Since the discovery of neutrino oscillations in 1998 [1] a considerable amount of progress has been made in understanding their mixing via solar, atmospheric, accelerator, and reactor neutrino measurements. Today it is known that there are transitions between all active neutrino flavors and the majority of parameters in the standard paradigm, known as the PMNS [2, 3] framework, have been measured. That being said there remain several open questions in the study of oscillations, the most prominent of which are the ordering of the neutrino mass states, the so-called neutrino "mass hierarchy" problem, whether or not neutrino oscillations are CP-violating, and the precise value of the "atmospheric" mixing angle, $\theta_{23}$. The present work focuses on the efforts of current and future experiments to address each of these issues. For the most part we will place emphasis on the experimental program in Japan, but parts of the global neutrino program will be mentioned in brief. It should be noted that though there are a diverse set of technologies and techniques being used throughout the global program, the present work is focused on atmospheric and accelerator neutrino measurements.

The present manuscript is organized as follows. A short review of PMNS oscillations is presented in Section 1 and is followed by discussions of atmospheric mixing parameter measurements and constraints on leptonic CP violation by present experiments in Sections 2 and 3. Prospects for future measurements are presented in Section 4 before concluding in Section 5.

\section{Neutrino Oscillations}

The term neutrino oscillation refers to the phenomenon of flavor change during neutrino propagation due to mixing between the neutrino mass $\left(v_{1}, v_{2}, v_{3}\right)$ and flavor eigenstates $\left(v_{e}, v_{\mu}, v_{\tau}\right)$. A unitary mixing matrix, known as the PMNS matrix [2,3], forms the bridge between two, $\left|v_{\alpha}\right\rangle=\sum_{i=1}^{3} U_{\alpha, i}^{*}\left|v_{i}\right\rangle$. This matrix is parameterized by three angles, $\theta_{i j}$, each representing a rotation between two states, which leads to the following decomposition:

$$
U=\left(\begin{array}{ccc}
1 & 0 & 0 \\
0 & c_{23} & s_{23} \\
0 & -s_{23} & c_{23}
\end{array}\right)\left(\begin{array}{ccc}
c_{13} & 0 & s_{13} e^{-i \delta_{c p}} \\
0 & 1 & 0 \\
-s_{13} e^{i \delta_{C P}} & 0 & c_{13}
\end{array}\right)\left(\begin{array}{ccc}
c_{12} & s_{12} & 0 \\
-s_{12} & c_{12} & 0 \\
0 & 0 & 1
\end{array}\right)
$$

where $c_{i j}\left(s_{i j}\right)$ indicates $\cos \theta_{i j}\left(\sin \theta_{i j}\right)$. Here the parameter $\delta_{c p}$ controls the CP behavior of the oscillations, with $\sin \delta_{c p} \neq 0$ inducing $\mathrm{CP}$ violation. There are three independent mass differences which enter the evolution equations for neutrinos, $\Delta m_{i j}^{2} \equiv m_{i}^{2}-m_{j}^{2}$, where $m_{i}$ labels the mass of $v_{i}$. It should be noted that the "1-2" and "2-3" mixing parameters are often referred to as the "solar" and "atmospheric" parameters as the oscillations they induce were first observed in atmospheric(c.f. [1]) and solar neutrino experiments(c.f. [4]). Modern long-baseline accelerator neutrino experiments, such as those discussed below, typically have sensitivity to the atmospheric and " $1-3$ " parameters of the matrix.

The probability of a neutrino of flavor $\alpha$ oscillating into flavor $\beta$ in vacuum can be written concisely as

$$
P\left(v_{\alpha} \rightarrow v_{\beta}\right)=\delta_{\alpha \beta}-4 \sum_{i>j} \Re\left\{U_{\alpha i}^{*} U_{\beta i} U_{\alpha j} U_{\beta j}^{*}\right\} \sin ^{2}\left(\frac{\Delta m_{i j}^{2} L}{4 E_{v}}\right)
$$




$$
+2 \sum_{i>j} \mathfrak{I}\left\{U_{\alpha i}^{*} U_{\beta i} U_{\alpha j} U_{\beta j}^{*}\right\} \sin \left(\frac{\Delta m_{i j}^{2} L}{2 E_{v}}\right) .
$$

Here $L$ and $E$ refer to the neutrino travel length and energy respectively. Oscillations in matter are complicated by the additional interaction of neutrinos with electrons in the media. Indeed, electron neutrinos undergo charged current coherent scattering, which is not felt by the other flavors, and thereby gives rise to an additional effective potential that is a function of the local electron density. For arbitrary matter profiles it is not possible to describe the oscillation probability in a simple closed form, but piecewise-constant profiles can be treated numerically [7].

It should be noted that since only $\Delta m_{i j}^{2}$ appears in Equation 1.2, oscillations are not sensitive to the absolute scale of the neutrino masses; only the differences in the mass states. For this reason the ordering of the neutrino mass states is unknown and at present there are only two possibilities, $m_{1}<m_{2} \ll m_{3}$ and $m_{3} \ll m_{1}<m_{2}$, which are referred to as the "normal" and "inverted" mass orderings respectively. The nature of the neutrino mass hierarchy is of interest since it induces different oscillations in matter for neutrinos and antineutrinos and thereby produces effects similar to intrinsic the CP effect of $\delta_{c p}$. In addition it has important connections to searches for neutrinoless double-beta decay [5] and theories of grand unification [6].

On further study of Equations 1.1 and 1.2 it is clear that neutrino oscillation measurements can only access $\delta_{c p}$ if all of the other mixing parameters are non-zero. Fortunately, this is the case (c.f. [5]) and, as is discussed further below, current experiments are now making the first constraints on this parameter. In addition, present data suggest that $\theta_{23}$ is $\sim 45^{\circ}$, known as "maximal mixing", but whether or not it differs significantly from this value is an open question.

In the present work we are mostly focused on long-baseline and atmospheric neutrino experiments, whose typical neutrino energies are $O(1) \mathrm{GeV}$ (and up to several $\mathrm{TeV}$ ) along pathlengths of $O(100) \mathrm{km}$ (and up to $10^{4} \mathrm{~km}$ ) for the former (latter). Under these conditions the dominant sensitivity to the neutrino mass hierarchy is derived from matter effects over long baselines and sensitivity to $\delta_{c p}$ is maximized through study of the "appearance" channel, $v_{\mu} \rightarrow v_{e}$ oscillations. The atmospheric mixing parameters, on the other hand, are constrained through $v_{\mu} \rightarrow v_{\mu}$ oscillations the "disappearance" channel. Oscillation probabilities for both the disappearance and appearance channels for long-baseline experiments at $210 \mathrm{~km}$ and $810 \mathrm{~km}$ are shown in Figure 1.

Figure 2 shows the $v_{\mu} \rightarrow v_{e}$ oscillation probability for both atmospheric neutrinos and antineutrinos as a function of energy and zenith angle assuming the normal mass hierarchy. The feature present between 2 and $10 \mathrm{GeV}$ seen in only the neutrino figure is a result of matter-induced resonances [8,9]. Interestingly, under an inverted mass hierarchy this feature moves to the antineutrino channel. For this reason atmospheric neutrino experiments can determine the mass hierarchy by studying the rate of electron and anti-electron neutrino appearance in the detector. It should be noted that matter effects of this sort are also present in accelerator experiments, though their strength depends on the neutrino baseline.

\section{Atmospheric Mixing}

Though the atmospheric mixing parameters were first constrained by atmospheric neutrino experiments, the most constraining results on $\Delta m_{32}^{2}$ and $\sin ^{2} \theta_{23}$ come from long-baseline experiments. With prior knowledge of the approximate value of the former in particular, $\Delta m_{32}^{2} \sim$ 

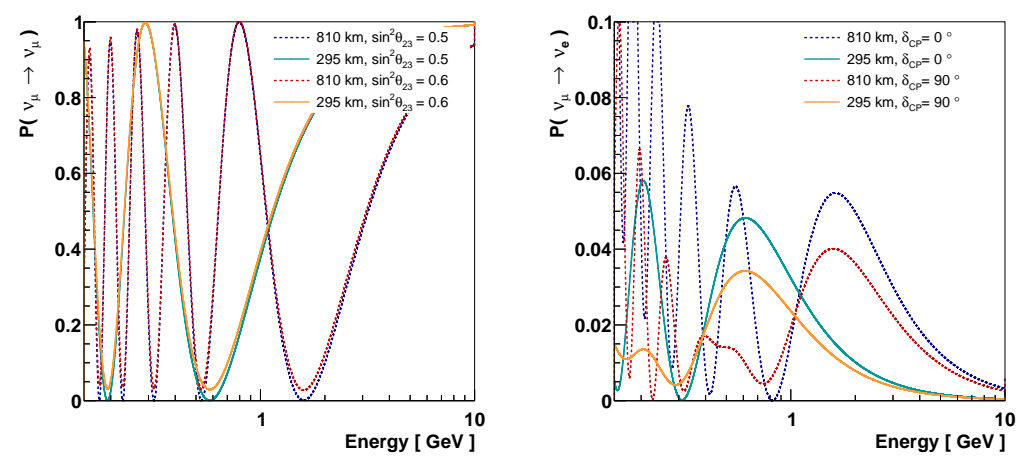

Figure 1: Neutrino oscillation probabilities as a function of energy for long-baseline experiments at $295 \mathrm{~km}$ and $810 \mathrm{~km}$. The left and right plots show the $v_{\mu} \rightarrow v_{\mu}$ and $v_{\mu} \rightarrow v_{e}$ oscillation probabilities for a normal mass hierarchy, respectively.
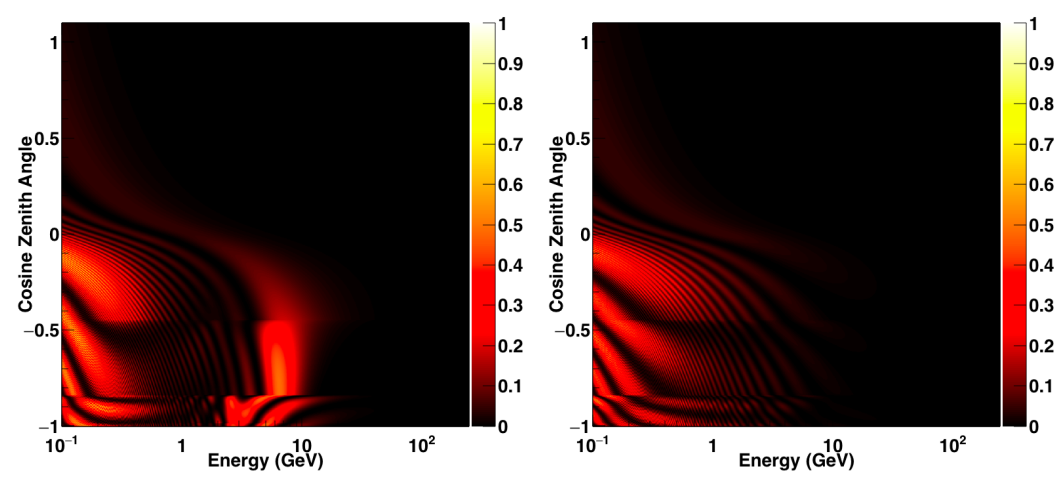

Figure 2: Neutrino oscillation probabilities as a function of zenith angle and energy. Cosine zenith of 1 $(-1)$ indicates downward-going (upward-going) neutrinos. The left and right plots illustrate the $v_{\mu} \rightarrow v_{e}$ and $\bar{v}_{\mu} \rightarrow \bar{v}_{e}$ oscillation probabilities assuming the normal mass hierarchy, respectively.
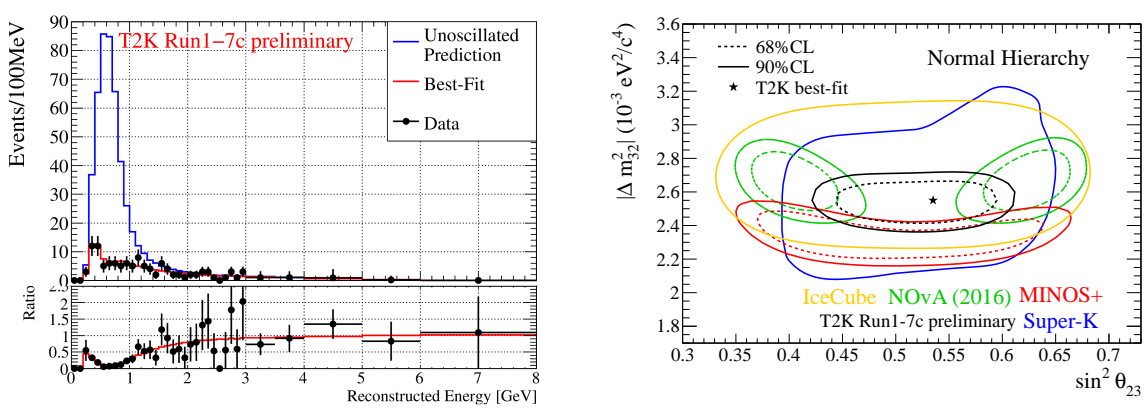

Figure 3: Disappearance data from the T2K experiment after an exponure of $7.48 \times 10^{20}$ protons on target with a neutrino enhanced beam are shown at left. The right plot shows constraints on the atmospheric mixing parameters for several experiments as of August, 2016 [10]. 
$2.5 \times 10^{3} \mathrm{eV}^{2}$, these experiments have optimized their sensitivity to the muon disappearance phenomenon by an appropriate choice of the $L / E$ appearing in Equation 1.2 as $\sim 450 \mathrm{~km} / \mathrm{GeV}$. While it is notoriously difficult to produce neutrino beams with monochromatic energies, the two experiments with leading sensitivity to atmospheric mixing, T2K [11] and NOvA [12], have located their detectors off of the central axis of their neutrino beams to achieve a sharply peaked neutrino flux. To observe the maximal oscillation effect (c.f. Figure 1) the T2K experiment's neutrino beam is directed $2.5^{\circ}$ (44 mrad) away from its far detector, Super-Kamiokande, which is located $295 \mathrm{~km}$ downstream of the production target. Similarly, the NOvA experiment is $0.8^{\circ}$ (14 mrad) off-axis from its beam's center, and located $810 \mathrm{~km}$ from the neutrino production point. At T2K the flux peaks at $\sim 650 \mathrm{MeV}$ and for NOvA at $\sim 2 \mathrm{GeV}$ in these configurations.

The power of this strategy is clearly illustrated in the left panel of Figure 3, which shows muon disappearance data from the $\mathrm{T} 2 \mathrm{~K}$ experiment in stark disagreement with the expectation assuming no oscillations. In the lower panel of the figure the ratio of the best fit oscillation hypothesis to the null oscillation hypothesis is shown. Roughly speaking the depth and energy of the nadir provide constraints on $\sin ^{2} \theta_{23}$ and $\Delta m_{32}^{2}$, respectively. This phenomenon is also present in the NOvA disappearance data (c.f. Figure 2 of Ref. [12]).

Recent atmospheric mixing constraints from the NOvA and T2K experiments are overlaid with other measurements, including atmospheric neutrino measurements at Super-Kamiokande [13] and IceCube [14], in the right panel of Figure 3. Like the long-baseline experiments these measurements rely on the disappearance of muon neutrinos. However, since the creation point and therefore pathlength of atmospheric neutrinos is not known a-priori, these experiments observe a suppression of the muon neutrino event across several reconstructed energies and zenith angles. The majority of the effect appears in the upward-going data due to the longer pathlength to the detector for typical atmospheric neutrino energies. Super-K observes these distortions down to $200 \mathrm{MeV}$ and while the lower energy threshold of IceCube is only $O(10) \mathrm{GeV}$, just on the tails of the oscillations, its larger size has yielded competitive constraints on the atmospheric mixing parameters. From the figure it is clear that the world's data agree well with $\Delta m_{32}^{2} \sim 2.5 \times 10^{3} \mathrm{eV}^{2}$ and $\sin ^{2} \theta_{23} \sim 0.5$. Interestingly, the NOvA data currently disfavor this precise value of the mixing angle by $2.6 \sigma$ [12], and are in slight tension with results from $\mathrm{T} 2 \mathrm{~K}$. Whether or not this hint will become evidence for non-maximal mixing is an exciting question to be address by future data sets.

\section{Electron Appearance: Mass Hierarchy and $\delta_{c p}$}

Of the experiments mentioned in the previous section Super-K, T2K, and NOvA presently have measurements of electron neutrino appearance which provide constraints on $\delta_{c p}$. For the long-baseline measurements the appearance of electron neutrino events via the $v_{\mu} \rightarrow v_{e}$ channel occurs at precisely the energy of their disappearance maximum. The amount of electron appearance is modulated by the value of $\delta_{c p}$, the mass hierarchy, the presence of matter effects, and the value of $\sin ^{2} \theta_{23}$. These experiments have the additional benefit of being able to produce either a predominantly neutrino or predominantly antineutrino beam and therefore directly test the difference between $v_{\mu} \rightarrow v_{e}$ and $\bar{v}_{\mu} \rightarrow \bar{v}_{e}$ oscillations to probe this parameter. Since the T2K baseline is short there is almost no matter-induced oscillation enhancement so this method provides a clean measurement of $\delta_{c p}$ with limited sensitivity to the mass hierarchy. NOvA, on the other hand, observes 

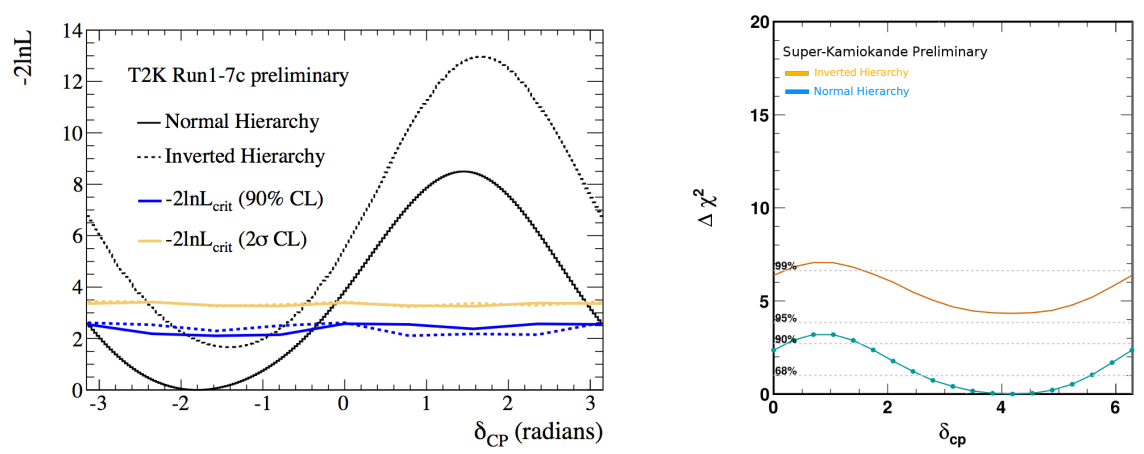

Figure 4: T2K's constraint on $\delta_{c p}$ for the normal (solid block) and inverted hierarchy assumptions (dashed black) with $7.48 \times 10^{20}\left(7.47 \times 10^{20}\right)$ protons on target with a neutrino (antineutrino) enhanced beam appears in the left panel [10]. Constraints from the Super-K atmospheric neutrino data are shown in the right panel assuming both the normal (blue) and inverted hierarchies (orange) after a 328 kton-year exposure [15]. The offset in the lines in both plots denotes the difference in their minimum likelihood values. The normal hierarchy is favored by both experiments.

sizable matter effects over its longer baseline and therefore has sensitivity to both parameters. At present T2K has observed 32 electron appearance events with a neutrino enhanced beam and four events with an antineutrino enhanced beam with expectations ranging between 19.6 to 28.7 events and 6.0 to 7.7 events for $\delta_{c p}=[-\pi / 2,+\pi / 2]$ [16]. The slight excess (deficit) of events events in the neutrino (antineutrino) channel drives $\mathrm{T} 2 \mathrm{~K}$ to favor the point at $-\pi / 2$ and the normal hierarchy as shown in the left panel of Figure 4. As a result the CP conserving points 0 and $\pi$ are disfavored at $90 \%$ C.L.. Similarly, NOvA has observed 33 candidates against a background of $8.2 \pm 0.8$ (syst), which induces a weak preference for the normal hierarchy, rejecting the inverted hierarchy at $93 \%$ assuming $\sin ^{2} \theta_{23}<0.5$ for all values of $\delta_{c p}$ [17].

Atmospheric neutrino sensitivity to $\delta_{c p}$ is driven by electron neutrino appearance at energies below $1 \mathrm{GeV}$ (c.f. Figure 2). Though there are both neutrinos and antineutrinos in the atmospheric neutrino flux and a large amount of data at these energies, Super-Kamiokande has no way of discriminating neutrino from antineutrino interactions event-by-event. In addition, the poor correlation between the incoming neutrino and its outgoing lepton obscures the effects of this parameter. Recent constraints from Super-Kamiokande indicate a preference for $\delta_{c p} \sim-\pi / 2$ but with less power to reject the $\mathrm{CP}$ conserving hypotheses [15]. On the other hand, the presence of large matter effects in the atmospheric neutrino data at multi-GeV energies gives Super-Kamiokande sensitivity to the mass hierarchy. Though the neutrino flux at these energies is considerably reduced relative to lower energies, excesses in the data are driving a preference for the normal hierarchy. The preference corresponds to a difference in the minima of the two hierarchy fits of $\Delta \chi_{N H-I H}^{2}=-4.3$ as shown in the right panel of Figure 2. Assuming the inverted mass hierarchy, the probability of observing a normal hierarchy preference at more than this level ranges between 0.7 and $3.1 \%$ depending upon the value of $\sin ^{2} \theta_{23}$ [15].

Currently the NOvA, T2K, and Super-Kamiokande experiments all favor the normal mass hierarchy and are consistent with $\delta_{c p} \sim-\pi / 2$. Though these hints have interesting implications, 


\begin{tabular}{l|cccc}
\hline \hline Experiment & 2020 & 2025 & 2030 & 2035 \\
\hline Super-Kamiokande & 2.5 & 3.0 & & \\
NOvA [19] & 3.4 & 4.4 & & \\
KM3NeT [21] & 0.5 & 4.0 & & \\
JUNO [22] & & 4.0 & & \\
ICAL-INO [23] & & 2.0 & 3.0 & 4.0 \\
DUNE [25] & & 3.0 & 5.0 & 7.0 \\
Hyper-Kamiokande [24] & & & 4.0 & 6.0 \\
\hline \hline
\end{tabular}

Table 1: Approximate mass hierarchy sensitivity as a function of year for various current and planned experiments assuming $\sin ^{2} \theta_{23}>0.5$ and notional time lines as presented in their repsective planning documents.

the preferences are weak and definitive measurements are being pursued by the next generation of these experiments.

\section{Future Prospects}

Both current and future experimental efforts are now focusing on addressing the issues of the neutrino mass ordering and the CP nature of neutrino oscillations. In the short term, existing projects like T2K, NOvA and Super-Kamiokande, which are for the most part statistics limited in these measurements, will continue to acquire data and increase their sensitivity. Though T2K has been approved for a total exposure of $7.8 \times 10^{21}$ protons on target, in order to achieve $3 \sigma$ sensitivity to CP violation, a $1.7^{\circ}$ measurement of $\theta_{23}$, and a $1 \%$ measurement of $\Delta m_{32}^{2}$, an extended run of the experiment has been proposed [18]. In addition to acquiring $20 \times 10^{21}$ protons on target, the project seeks to improve neutrino interaction and flux systematic errors with an upgraded near detector and to increase the far detector sample by $50 \%$ through improved reconstruction techniques in order to meet these goals. NOvA is still taking data as part of its initial design but expects to determine the mass hierarchy at the $95 \%$ C.L. and reject maximal atmospheric mixing at more than $3 \sigma$ within three years [19]. By 2024 it will have $2 \sigma$ sensitivity to $\mathrm{CP}$ violation assuming the normal mass hierarchy and $\delta_{c p} \sim-\pi / 2$.

Upgrades to both the IceCube [20] and KM3NeT [21] neutrino telescopes to lower their energy thresholds to $O(1) \mathrm{GeV}$ are being planned to give these experiments better access to the neutrino mass hierarchy. In addition to atmospheric mixing sensitivity through the disappearance channel, these detectors can also distinguish muon and electron neutrino interactions at a level sufficient for $4.0 \sigma$ or more hierarchy sensitivity due to their megaton-size volumes. It should be noted that the muon channel alone can be used to determine the mass hierarchy, which is something that the ICAL-INO detector will take advantage of to obtain $4.0 \sigma$ sensitivity after a 1 Mton-year exposure [23]. Increases in Super-Kamiokande's hierarchy sensitivity are expected to come in the form of reconstruction improvements to utilize more of the detector's target volume and to reduce backgrounds such as oscillation-induced $v_{\tau}$ interactions. Table 1 summarizes the approximate hierarchy sensitivity of these and other experiments for the next 20 years. 
In order to achieve sensitivity to $\mathrm{CP}$ violation beyond $3 \sigma$ the next generation of experiments is necessary. In addition to searches for proton decay and studies of astrophysical neutrinos, the Hyper-Kamiokande [24] and DUNE [25] experiments are being designed for this purpose. As the name suggests, Hyper-Kamiokande is the successor to the Super-Kamiokande and T2K experiments and will combine atmospheric neutrino and long-baseline measurements to obtain maximum sensitivity to neutrino oscillations. Two 187 kton detectors (one of which may be located in Korea [26]) with high quantum efficiency photodetectors and a 1.3 MW beam are anticipated. After a 10 year exposure Hyper-Kamiokande can observe $\mathrm{CP}$ violation at more than $5 \sigma$ for more than $60 \%$ of $\delta_{c p}$ phase space. Further, it is expected to measure the atmospheric mixing parameters to $O(1) \%$, to determine the mass hierarchy at $5 \sigma$, and to observe non-maximal mixing at more than $3 \sigma$ provided $\left|\theta_{23}-45^{\circ}\right|>3^{\circ}$. Hyper-Kamiokande's sensitivity is summarized in Figure 5 . DUNE expects to have complementary sensitivity, with $5 \sigma$ sensitivity to CP violation for $50 \%$ of $\delta_{c p}$ phase space after a 550 kton-MW-year exposure [25]. Considering the lengthy time scales for the construction of these detectors, between 7 and 10 years, a definitive measurement of $\delta_{c p}$ is likely 20 years in the future.
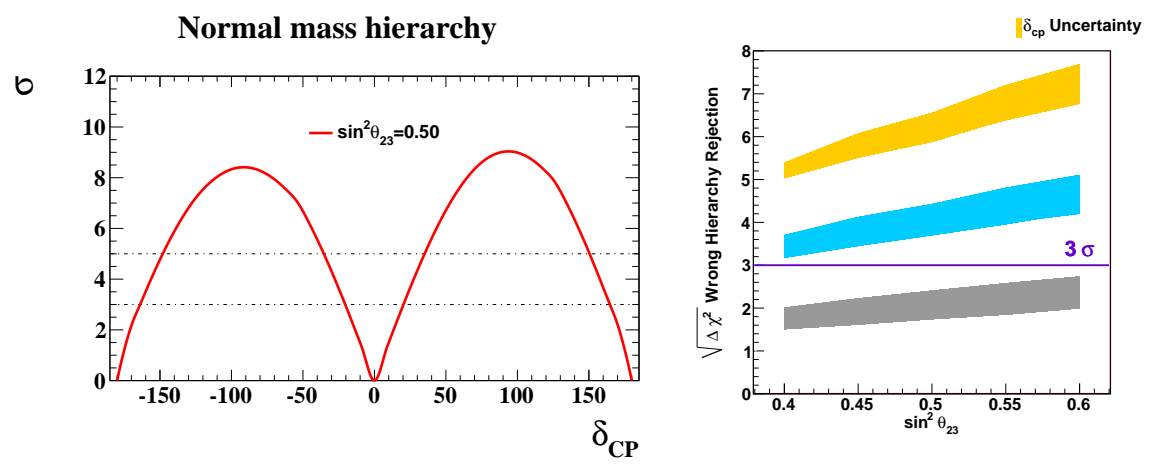

Figure 5: Hyper-Kamiokande's sensitivity to $\mathrm{CP}$ violation after 10 years is shown in the left panel. The right panel shows the sensitivity to the mass hierarchy after one (grey), five (blue), and 10 years (gold) of data. The width of the bands represents the uncertainty from $\delta_{c p}$.

\section{Conclusions}

Considerable progress has been made in the understanding of neturino oscillations since the start of the industry nearly 20 years ago. Indeed, all of the mixing angles and mass differences in the standard PMNS framework have been measured and several experimental efforts are now focused on understanding the neutrino mass hierarchy, the CP nature of these oscillations, and whether or not atmospheric mixing is maximal. At present the global data weakly favor a normal mass hierarchy and $\delta_{c p} \sim \pi / 2$ but whether or not these hints persist is at the center of planning efforts for the next generation of experiments. Though the funding scenarios for those projects are currently uncertain, the realization of only a handful of the possible technologies would contribute to the resolution of the mass hierarchy and a $3 \sigma$ or better observation of CP violation in the next 10 to 20 years. 


\section{References}

[1] Y. Fukuda et al. [Super-Kamiokande Collaboration], Phys. Rev. Lett. 81, 1562 (1998) doi:10.1103/PhysRevLett.81.1562 [hep-ex/9807003].

[2] B. Pontecorvo, Sov. Phys. JETP 26, 984 (1968) [Zh. Eksp. Teor. Fiz. 53, 1717 (1967)].

[3] Z. Maki, M. Nakagawa and S. Sakata, Prog. Theor. Phys. 28, 870 (1962). doi:10.1143/PTP.28.870

[4] Q. R. Ahmad et al. [SNO Collaboration], Phys. Rev. Lett. 87, 071301 (2001) doi:10.1103/PhysRevLett.87.071301 [nucl-ex/0106015].

[5] C. Patrignani et al. [Particle Data Group], Chin. Phys. C 40, no. 10, 100001 (2016). doi:10.1088/1674-1137/40/10/100001

[6] R. N. Mohapatra et al., Rept. Prog. Phys. 70, 1757 (2007) doi:10.1088/0034-4885/70/11/R02 [hep-ph/0510213].

[7] V. D. Barger, K. Whisnant, S. Pakvasa and R. J. N. Phillips, Phys. Rev. D 22, 2718 (1980). doi:10.1103/PhysRevD.22.2718

[8] E. K. Akhmedov, Sov. J. Nucl. Phys. 47, 301 (1988) [Yad. Fiz. 47, 475 (1988)].

[9] P. I. Krastev and A. Y. Smirnov, Phys. Lett. B 226, 341 (1989). doi:10.1016/0370-2693(89)91206-9

[10] K. Iwamoto [T2K Collaboration], PoS ICHEP 2016 (2016).

[11] K. Abe et al. [T2K Collaboration], Nucl. Instrum. Meth. A 659, 106 (2011) doi:10.1016/j.nima.2011.06.067 [arXiv:1106.1238 [physics.ins-det]].

[12] P. Adamson et al. [NOvA Collaboration], [arXiv:1701.05891 [hep-ex]].

[13] K. Abe et al., Nucl. Instrum. Meth. A 737, 253 (2014) doi:10.1016/j.nima.2013.11.081 [arXiv:1307.0162 [physics.ins-det]].

[14] R. Abbasi et al. [IceCube Collaboration], Astropart. Phys. 35, 615 (2012) doi:10.1016/j.astropartphys.2012.01.004 [arXiv:1109.6096 [astro-ph.IM]].

[15] S. Moriyama [Super-Kamiokande Collaboration], “27th International Conference on Neutrino Physics and Astrophysics (Neutrino 2016)"

[16] K. Abe et al. [T2K Collaboration], arXiv:1701.00432 [hep-ex].

[17] P. Adamson et al. [NOvA Collaboration], arXiv:1703.03328 [hep-ex].

[18] K. Abe et al., arXiv:1609.04111 [hep-ex].

[19] M. Strait [NOvA Collaboration], Presentation at MIAMI 2016, http://nova-docdb.fnal.gov/cgi-bin/ShowDocument?docid=16687

[20] M. G. Aartsen et al. [IceCube Collaboration], arXiv:1607.02671 [hep-ex].

[21] S. Adrian-Martinez et al. [KM3Net Collaboration], J. Phys. G 43, no. 8, 084001 (2016) doi:10.1088/0954-3899/43/8/084001 [arXiv:1601.07459 [astro-ph.IM]].

[22] F. An et al. [JUNO Collaboration], J. Phys. G 43, no. 3, 030401 (2016) doi:10.1088/0954-3899/43/3/030401 [arXiv:1507.05613 [physics.ins-det]].

[23] S. Ahmed et al. [ICAL Collaboration], arXiv:1505.07380 [physics.ins-det].

[24] [Hyper-Kamiokande Collaboration], KEK-PREPRINT-2016-21, ICRR-REPORT-701-2016-1.

[25] R. Acciarri et al. [DUNE Collaboration], arXiv:1512.06148 [physics.ins-det].

[26] K. Abe et al. [Hyper-Kamiokande proto-Collaboration], arXiv:1611.06118 [hep-ex]. 